\title{
Neighborhood crime is differentially associated with cardiovascular risk factors as a function of race and sex
}

\author{
Mollie R. Sprung, ${ }^{1,2}$ Lauren M.D. Faulkner, ${ }^{2}$ Michele K. Evans, ${ }^{3}$ Alan B. Zonderman, ${ }^{3}$ \\ Shari R. Waldstein ${ }^{2}$ \\ ${ }^{1}$ Veterans Affairs, Pittsburgh Healthcare System, University Drive, Pittsburg, PA; ${ }^{2}$ Department of \\ Psychology, University of Maryland, Baltimore County, Baltimore, MD; ${ }^{3}$ Laboratory of Epidemiology and \\ Population Sciences, National Institute on Aging, Baltimore, MD, USA
}

Significance for public health

Neighborhood crime may be an important factor contributing to cardiovascular health disparities, and these relations may vary by race and sex. Among 1,718 AAs and Whites (58\% AA; 54\% female; $59 \%$ above poverty; ages 30-64 years) living Baltimore, Maryland who completed the first wave of the Healthy Aging in Neighborhoods of Diversity across the Life Span study from 2004-2009, greater overall crime rate was related to higher fasting glucose levels, and greater violent crime rate was associated with higher systolic and diastolic blood pressure in AA women, but not men. No associations were found in Whites. Results indicate that AA women may be particularly vulnerable to the negative impact of crime on cardiovascular risk. Preventative efforts aimed toward this group may help to deter the detrimental effects that living in a high crime area may have on one's cardiovascular health.

\section{Abstract}

Background: Neighborhood crime may be an important factor contributing to cardiovascular health disparities, and these relations may vary by race and sex. The present investigation evaluated (a) potential differential associations between neighborhood crime and cardiovascular disease (CVD) risk factors within subgroups of African American (AA) and White men and women, and (b) potential mediation by negative affect.

Design and Methods: Participants were 1,718 AAs and Whites (58\% AA; 54\% female; 59\% above poverty; ages 30-64 years) living Baltimore, Maryland who completed the first wave of the Healthy Aging in Neighborhoods of Diversity across the Life Span study from 2004-2009. CVD risk factors included body mass index, total serum cholesterol, glucose, and systolic and diastolic blood pressure. A negative affect composite was comprised of self-reported depression, anxiety, anger, vigilance, and perceived stress. Hierarchical multiple regression analyses were used to examine associations between per capita overall and violent crime rates, negative affect, and CVD risk factors.

Results: There were significant associations of greater overall crime rate with higher fasting glucose $(b=192, \mathrm{P}<0.05)$, and greater violent crime rate with higher systolic $(b=86.50, \mathrm{P}<0.05)$ and diastolic $(b=60.12, \mathrm{P}<0.05)$ blood pressure in AA women, but not men. These associations were not explained by negative affect. In Whites, there were no significant associations of overall or violent crime rates with cardiovascular risk factors

Conclusions: AA women may be particularly vulnerable to the negative impact of crime on cardiovascular risk. Preventative efforts aimed toward this group may help to deter the detrimental effects that living in a high crime area may have on one's cardiovascular health.

\section{Introduction}

Cardiovascular disease (CVD) is the leading cause of death and disability in the United States for both men and women. ${ }^{1}$ During the past several decades, research has established an association between lower socioeconomic status (SES) and greater CVD morbidity and mortality. ${ }^{2}$ Individual SES factors such as poverty are often geographically concentrated. ${ }^{3}$ Theories suggest that the environmental context within which one lives may play an important role in both defining and maintaining one's SES. ${ }^{3,4}$ Indeed, physical and social dimensions of neighborhood are associated with CVD risk factors independent of individual SES. ${ }^{5-12}$ However, this research has examined mostly composite measures of neighborhood characteristics, whereas findings based on specific factors may be more directly translatable to intervention and prevention efforts.

Within the limited available research on specific neighborhood factors, considerable attention has been given to crime exposure, which has been associated with CVD risk factors and morbidity as well as various dimensions of negative affect. ${ }^{13-19}$ CVD risk factors associated with crime exposure include greater blood pressure, heart rate, pulse rate reactivity, body mass index, metabolic syndrome (including elevated waist circumference and fasting glucose), triglyceride levels, C-reactive protein, low-density lipoprotein (LDL) cholesterol, and a composite measure of CHD risk including reduced high-density (HDL) and elevated total cholesterol. ${ }^{15-18,20-31}$ Much of this research, however, examined crime exposure within children or adolescents, or homogeneous samples that are not generalizable to other populations.

Previous literature suggests that Blacks and Whites are differentially exposed to crime and that men and women have differential reactions to crime. ${ }^{32-34}$ Likewise, CVD risk factors vary by race and sex. ${ }^{35-39}$ These observations suggest that the associations between crime and CVD risk factors may differ across race and sex; an issue neglected in the literature thus far. Additionally, much of the literature has used only subjective self-reports of crime exposure or perceived safety. Information on crime prevalence collected by local government and police departments offer more objective measures of crime and may thus allow for more accurate implications of findings.

Because research has revealed associations of negative affect with both CVD and crime exposure, it may at least partially medi- 
ate the relation of the sociophysical environment to CVD. ${ }^{40-43}$ Several studies have examined associations among individuallevel SES, negative affect, and CVD variables, but offer conflicting findings. ${ }^{42,44-46}$ To our knowledge, only one study has examined sociophysical neighborhood factors, negative affect, and health outcome variables in the same statistical model. In that regard, Hill et al. ${ }^{41}$ found that associations between a composite measure of neighborhood disorder (that included crime) and general health were mediated by depression and anxiety. To our knowledge, no study has examined whether measures of negative affect mediate the association between crime exposure and CVD or its risk factors.

The present study examined the association between objective crime exposure and risk factors for CVD within a racially and socioeconomically heterogeneous adult sample. We hypothesized that higher levels of neighborhood crime are associated with more pronounced CVD risk factors after adjusting for individual-level SES and other socio-demographic risk factors, behavioral factors, and medical comorbidities. We further hypothesized that this association is at least partially mediated by a composite measure of negative affect and perceived stress. Lastly, we explored whether associations might differ by race and sex.

\section{Materials and Methods}

\section{Participants}

Data for this study were derived from the first wave of the Healthy Aging in Neighborhoods of Diversity across the Life Span (HANDLS) study performed by the National Institute on Aging's Intramural Research Program. ${ }^{47}$ HANDLS is an ongoing longitudinal investigation of race and SES- related health disparities among AA and White adults aged 30-64 years residing in 13 Baltimore neighborhoods pre-selected based on their likelihood to yield representative distributions of race and sex across a range of socioeconomic circumstances. ${ }^{42,47}$ A total of 2,800 participants completed both phases of the first wave of the study which took place from 2004-2009.

Medical exclusions for the study were individuals who were: 1) currently pregnant; 2) within 6 months of receiving chemotherapy, radiation, or biological treatments for cancer; 3) diagnosed with AIDS; 4) unable to provide informed consent; 5) currently using illegal/illicit drugs; or 6) with uncontrolled high blood pressure $(>160 / 100)$. Please refer to previous publications for additional study exclusions. ${ }^{47}$ The current analyses further excluded individuals: 1) who did not complete all psychosocial measures $(\mathrm{n}=586) ; 2)$ with a history of stroke $(\mathrm{n}=60)$, dialysis $(\mathrm{n}=1)$, dementia $(n=4)$, or cancer $(n=250) ; 3)$ with a current diagnosis of chronic or severe cognitive or psychiatric conditions including schizophrenia $(n=39)$ and bipolar disorder $(n=128) ; 7)$ with a current diagnosis of neurological conditions including Parkinson's Disease $(n=3)$, Alzheimer's Disease $(n=0)$, epilepsy $(n=99)$, and multiple sclerosis $(n=5)$; or 6) who were not fasting at the time their labs were drawn $(n=178)$. After these additional exclusions, 1,718 of the 2,800 participants remained.

Participants were identified during the first phase of the study by trained field interviewers who went door-to-door to gather demographic information. Based on an area probability sample, randomly selected residents were invited to participate in the study and, upon informed consent, were administered a household and nutritional survey, which included self-reported demographic questions. The second phase took place on a Medical Research
Vehicle (MRV) which visited each of the 13 neighborhoods. During this visit, medical personnel conducted a medical history and physical exam, and administered a series of psychological assessments.

\section{Ethics approval and consent to participate}

All authors have complied with the Principles of the Ethical Practice of Public Health of APHA. The registration number of the Healthy Aging in Neighborhoods of Diversity across the Life Span (HANDLS) study is NCT01323322. The HANDLS study was approved by the Institutional Review Board of the National Institute of Environmental Health Sciences, NIH. All participants provided written informed consent.

\section{Availability of data and material}

Crime data were made available by the Baltimore Neighborhood Indicators Alliance-Jacob France Institute of the University of Baltimore. HANDLS data are available through reasonable request; see https://handls.nih.gov/

\section{Measures \\ Demographic variables}

Participants self-reported their race $(1=\mathrm{AA}, 0=$ White $)$, sex ( $1=$ men, $0=$ women), education (years), and length of residence (years in current home). Poverty status was characterized as a family income less than $125 \%$ of the Federal poverty threshold and coded as 1, "poverty" and those with a family income greater than or equal to $125 \%$ of the poverty threshold were coded as 0 , "not poverty."

\section{Characterization of exposure to crime}

The HANDLS study used 2000 census tract data to define its 13 neighborhoods as contiguous groups of tracts. Crime statistics were made available by the Baltimore Neighborhood Indicators Alliance - Jacob France Institute of the University of Baltimore which provided the total number of overall and violent crime events for 2000 census tract neighborhoods from 2000-2008. Overall crime was defined as incidents of homicide, rape, aggravated assault, robbery, burglary, larceny, and auto theft that were reported to the Police Department. Violent crime was defined as the number of overall crimes identified as being violent (homicide, rape, aggravated assault, and robbery) that were reported to the Police Department. Cumulative overall and violent per capita crime rates were calculated for each participant by summing annual crime rates (total annual crime per capita based on 2000 population) for their census tract of residence for the four years before their year of enrollment.

\section{Behavior/lifestyle factors}

Alcohol and drug status were determined such that $0=$ not current user (includes never tried, never used regularly, and used $>6$ months ago) and $1=$ used within the last 6 months. Smoking status was categorized such that $0=$ not current smoker (includes never smoked, experimented with $<100$ cigarettes, and former smoker) and $1=$ current smoker.

\section{Psychological factors}

Perceived stress was measured by Cohen's 4-item Perceived Stress Scale and depressive symptoms were measured by the Center for Epidemiological Studies Depression Scale (CESD). ${ }^{48,49}$ The generalized anxiety disorder subscale of the Psychiatric Diagnostic Screening Questionnaire (PDSQ) was used 
to measure anxiety. ${ }^{50}$ Vigilance, as characterized by the need to chronically monitor the environment for threatening cues, was measured by a subscale of the Multiscale Measure of Reactive Responding, and anger was measured by a subscale of the Buss and Perry Aggression Questionnaire. ${ }^{51,52}$

\section{Anthropometric measures}

Body mass index (BMI) was calculated for each participant by dividing their measured weight by their measured height $\left(\mathrm{kg} / \mathrm{m}^{2}\right)$.

\section{Comorbidity covariates}

Data on potentially confounding premorbid medical conditions were grouped into clusters. Diabetes mellitus, thyroid disease, kidney disease, hepatitis, lupus, and HIV/AIDS were included in the diabetes mellitus, metabolic, and infectious disorders (DMMI) cluster. History of coronary artery disease, myocardial infarction, peripheral artery disease, atrial fibrillation, angioplasty, carotid endarterectomy, and coronary artery bypass surgery were included in the cardiovascular disease (CVD) cluster. All medical conditions were dichotomized. The cluster variables were calculated by summing these scores across conditions within the clusters. Additionally, BMI was used as a covariate when it was not used as a dependent variable. Use of psychotropic and anti-hypertensive medications were included as covariates for all analyses and use of statins was included as a covariate for the TSC analyses

\section{Metabolic measures}

Fasting blood samples were obtained for assessment of total serum cholesterol (TSC)and glucose (GLU). TSC and GLU were derived spectrophotometrically.

\section{Hemodynamic measures}

The brachial artery auscultation method was used to measure systolic and diastolic blood pressure (SBP, DBP). Measures were obtained with the arm at a 90-degree angle, palm facing up. One measure was obtained in each arm; these measures were averaged for analyses.

\section{Data analyses}

Missingness was examined for all of the variables. Data for all of the dependent variables except BMI (no missing data) were imputed with predicted values based on regression estimations using age, sex, race, and poverty status. These variables all had less than $10 \%$ missing by sex, race, and poverty status subgroups. The only remaining variables that had missing values were education $(n=42)$, length of residence $(n=377)$, and use of statins $(n=135)$.

Table 1. Mean values for sample characteristics and all variables by race and sex.

\begin{tabular}{|c|c|c|c|c|c|c|}
\hline \multirow[t]{2}{*}{ Variable } & \multicolumn{2}{|c|}{ White $(n=716)$} & \multicolumn{2}{|c|}{ Black $(n=1002)$} & \multicolumn{2}{|c|}{ Overall ( $n=1718$ ) } \\
\hline & Male & Female & Male & Female & Male & Female \\
\hline Age (years) & 48.12 & 47.19 & 47.62 & 47.12 & 47.82 & 47.15 \\
\hline Education (years) & 13.20 & 12.74 & 12.34 & 12.34 & 12.68 & 12.50 \\
\hline$\%$ Poverty $^{\mathrm{a}}$ & 25 & 34 & 47 & 51 & 38 & 44 \\
\hline Length of Residence (years) & 12.78 & 12.76 & 10.17 & 10.29 & 11.28 & 11.34 \\
\hline Overall Crime Rate (per capita) ${ }^{b}$ & 0.30 & 0.30 & 0.29 & 0.29 & 0.30 & 0.30 \\
\hline Violent Crime Rate (per capita) ${ }^{c}$ & 0.07 & 0.07 & 0.08 & 0.08 & 0.08 & 0.08 \\
\hline Anxiety ${ }^{d}$ & 2.36 & 3.51 & 2.35 & 2.87 & 2.35 & 3.14 \\
\hline CES-De & 12.33 & 15.04 & 13.52 & 14.35 & 13.03 & 14.64 \\
\hline Perceived Stress Scale ${ }^{f}$ & 5.35 & 5.74 & 5.27 & 5.16 & 5.31 & 5.41 \\
\hline Anger ${ }^{\mathrm{g}}$ & 10.80 & 10.22 & 8.97 & 9.71 & 9.72 & 9.92 \\
\hline Vigilance $^{\mathrm{h}}$ & 11.02 & 11.17 & 11.62 & 11.65 & 11.37 & 11.45 \\
\hline CVHTN $^{\mathrm{i}}$ & 0.12 & 0.15 & 0.19 & 0.16 & 0.16 & 0.15 \\
\hline DMMIj $^{j}$ & 0.25 & 0.32 & 0.36 & 0.31 & 0.32 & $0.3^{2}$ \\
\hline \% Psychotropic Medication Use & 13 & 19 & 7 & 10 & 9 & 14 \\
\hline \% Antihypertensive Medication Use & 26 & 21 & 25 & 35 & 25 & 29 \\
\hline \% Statin Use & 17 & 13 & 8 & 12 & 12 & 13 \\
\hline \% Current Drug Use & 15 & 8 & 26 & 13 & 22 & 11 \\
\hline$\%$ Current Alcohol Use & 63 & 47 & 61 & 48 & 62 & 48 \\
\hline \% Current Smoker & 44 & 38 & 55 & 40 & 50 & 39 \\
\hline Glucose (mg/dL) & 110.61 & 105.05 & 103.87 & 101.37 & 106.66 & 102.91 \\
\hline Systolic Blood Pressure (mmHg) & 121.10 & 115.28 & 121.52 & 121.49 & 121.35 & 118.89 \\
\hline Diastolic Blood Pressure (mmHg) & 74.69 & 69.86 & 74.56 & 72.33 & 74.61 & 71.29 \\
\hline Body Mass Index $\left(\mathrm{kg} / \mathrm{m}^{2}\right)$ & 28.96 & 31.07 & 27.43 & 31.87 & 28.06 & 8.67 \\
\hline Total Serum Cholesterol (mg/dL) & 186.81 & 195.78 & 180.56 & 188.03 & 183.15 & 191.28 \\
\hline
\end{tabular}

CES-D = Center for Epidemiological Studies Depression Scale; CVHTN = Cardiovascular disease cluster; DMMI = Diabetes mellitus, metabolic, and infectious disorders cluster; LDL= low density lipoprotein; HDL= high density lipoprotein. aPoverty defined as family income less than $125 \%$ of the Federal poverty threshold. ${ }^{b}$ Overall Crime Rate defined as the sum of annual per capita overall criminal offenses (murder, aggravated assault, rape and attempted rape, robbery, burglary, larceny, and auto theft) for census tract of residence for 4 years prior to enrollment. cViolent Crime Rate defined as the sum of annual per capita violent criminal offenses (murder, rape and attempted rape, aggravated assault, robbery) for census tract of residence for 4 years prior to enrollment. dAnxiety defined as score on generalized anxiety disorder subscale of the Psychiatric Diagnostic Screening Questionnaire. ${ }^{39}$ eCESD38 is a standard measure of depressive symptoms. Perceived stress defined as score on Cohen's 4-item Perceived Stress Scale. ${ }^{37}$ gAnger defined as score on anger subscale of Buss and Perry Aggression Questionnaire. ${ }^{41}$ hVigilance defined as score on vigilance subscale of Multiscale Measure of Reactive Responding. ${ }^{40}$ iCVHTN defined as number of cardiovascular disease conditions including coronary artery disease, myocardial infarction, peripheral artery disease, atrial fibrillation, angioplasty, carotid endarterectomy, and coronary artery bypass surgery. "higilance defined as score on vigilance subscale of Multiscale Measure of Reactive Responding. ${ }^{40}$ jDMMI defined as number of diabetes mellitus, metabolic, and infectious disorders including thyroid disease, kidney disease, hepatitis, lupus, and HIV/AIDS. 
Given the large sample size, regressions were run using listwise deletion to account for this missing data. Variables were also examined for normality. Length of residence, TSC, and glucose were found to be non-normal and were $\log 10$ transformed in efforts to normalize distributions.

Since previous findings have suggested that psychosocial stressors tend to cluster together and that their combined effect may lead to even higher risk for cardiovascular events, it was expected that perceived stress, vigilance, anger, anxiety, and depression would be closely related and would represent a cumulative emotional burden. ${ }^{53}$ A principle components analysis (PCA) of these measures indeed yielded one component extracted based on Kaiser-Guttman criterion. ${ }^{54}$ The component, on which factor loadings ranged from .56 to .82 , was used in the analyses to account for the five psychosocial measures.

We stratified rather than examined interactions of crime by race and sex because of the likelihood of differential confounding as well as differential mediation mechanisms by race and sex. After stratifying the sample by race and sex, we conducted a series of hierarchical multiple regressions to assess relations between overall crime rate, negative affect, and each CVD risk factor. The first step of the models included overall crime rate and demographic variables (age, education, poverty status, and length of residence) to determine the effect of overall crime rate on each cardiovascular risk factor after controlling for common covariates. The second step included both comorbidity clusters (CVD and DMMI) and medication use, and the third step included alcohol, drug, and smoking status as well as BMI (except for and BMI analyses) to determine if any associations between crime and cardiovascular risk factors remained after further adjustment. The fourth and final step included the negative affect component to examine potential mediation. A second series of identical hierarchical multiple regressions were conducted with violent crime rate. An $\alpha$ level of 0.05 was used to determine statistical significance.

\section{Results}

\section{Descriptive statistics}

Descriptive statistics for all variables by race and sex are provided in Table 1. The average per capita overall crime rate across all participants was .30 and the average per capita cumulative violent crime rate was .08. This means that on average, 300 overall crimes were reported per every 1000 residents living in a given participant's census tract during the 4 years before their enrollment in the HANDLS study, and approximately 80 of those crimes were coded as violent.

\section{Hierarchical regressions}

Results showed a main effect of overall crime on GLU and of violent crime on SBP and DBP in AA women. In AA women only, greater overall crime rate was associated with higher GLU $(b=.19$, $\left.t(420)=2.62, \mathrm{P}<.05, r^{2}=.014\right)$ and greater violent crime rate was associated with higher SBP $(b=86.504, t(420)=2.36, \mathrm{P}<.05$, $\left.r^{2}=.011\right)$ and DBP $\left(b=60.12, t(420)=2.49, \mathrm{P}<.05, r^{2}=.014\right)$. These associations were not attenuated by negative affect. In AA men and Whites, there were no significant associations of overall or violent crime rates with any of the cardiovascular risk factors (results not shown). Complete regression results are reported in Tables 2-4.

\section{Discussion}

We found that for AA women, living in an area of greater overall crime was associated with higher GLU, and living in an area of greater violent crime was associated with greater SBP and DBP. These findings were independent of select individual-level SES and other socio-demographic risk factors, behavioral factors, and medical comorbidities. That significant associations were only present for AA women suggests that this subset of the population may be particularly vulnerable to the health consequences of

Table 2. Hierarchical unstandardized regression coefficients for overall crime rate predicting glucose in AA women.

\begin{tabular}{|c|c|c|c|c|}
\hline & Step 1 & Step 2 & Step 3 & Step 4 \\
\hline Age & 0.001 & 0.000 & 0.000 & 0.000 \\
\hline Education & -0.001 & 0.000 & -0.001 & -0.001 \\
\hline Poverty Status ${ }^{\mathrm{a}}$ & -0.018 & -0.013 & -0.014 & -0.012 \\
\hline Length of Residence & 0.016 & 0.017 & 0.017 & 0.016 \\
\hline Overall Crime Rate ${ }^{b}$ & $0.174^{*}$ & $0.186^{*}$ & $0.182^{*}$ & $0.192^{*}$ \\
\hline CVHTN $^{\mathrm{c}}$ & & 0.016 & 0.016 & 0.017 \\
\hline DMMI $^{d}$ & & $0.023^{*}$ & $0.022^{*}$ & $0.024^{*}$ \\
\hline RxPsychotropic & & -0.011 & -0.013 & -0.006 \\
\hline RxHTN & & $0.034 *$ & $0.034 *$ & $0.035^{*}$ \\
\hline BMI & & $0.003^{* *}$ & $0.003^{* *}$ & $0.003^{* *}$ \\
\hline Current Drug & & & -0.016 & -0.014 \\
\hline Current Alcohol & & & -0.006 & -0.005 \\
\hline Current Smoking & & & 0.011 & 0.013 \\
\hline \multicolumn{4}{|l|}{ Negative Affect Component ${ }^{\mathrm{e}}$} & $-0.016^{*}$ \\
\hline \multicolumn{5}{|c|}{ 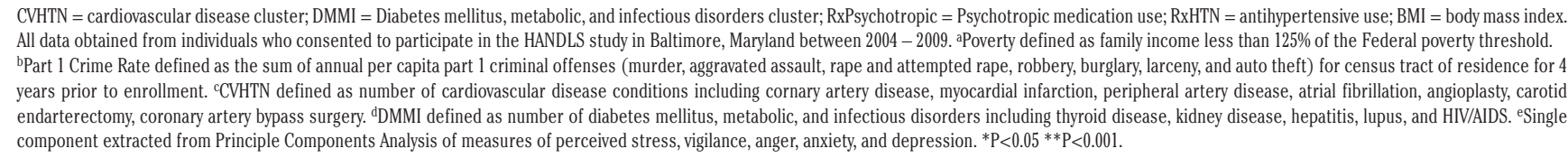 } \\
\hline
\end{tabular}


neighborhood crime. The results further suggest that blood pressure and glucose levels may be particularly sensitive to crime exposure among AA women, although the cross-sectional design of this study prevents us from making any inferences regarding temporal direction.

In support of the present results, previous findings suggest that neighborhood stressors may at least partially explain racial differences in hypertension prevalence, an important consequence of elevated blood pressure. ${ }^{36}$ Furthermore, in a study by Wilson et al., ${ }^{19}$ violence exposure was associated with elevated nighttime blood pressure among AA adolescents. Although there have been no direct examinations of crime and fasting glucose, Baldock et $a l .{ }^{23}$ found that the metabolic syndrome (including elevated fasting glucose) was associated with greater perceived neighborhood crime in a sample of middle-aged men and women. In another study, a short-term spike in burglary rates was associated with increased C-reactive protein (which has been positively associated with glucose levels), ${ }^{24}$ although associations were observed for men, but not women. ${ }^{55}$

Research has suggested that women may be particularly vul-

Table 3. Hierarchical unstandardized regression coefficients for violent crime rate predicting SBP in Black women.

\begin{tabular}{|c|c|c|c|c|}
\hline & Step 1 & Step 2 & Step 3 & Step 4 \\
\hline Age & $0.645^{* *}$ & $0.59^{* *}$ & $0.592^{* *}$ & $0.596^{* *}$ \\
\hline Education & $-0.786^{*}$ & $-0.793^{*}$ & $-0.799^{*}$ & $-0.781^{*}$ \\
\hline Poverty Status ${ }^{\mathrm{a}}$ & -0.363 & 0.362 & 0.490 & 0.459 \\
\hline Length of Residence & $4.505^{*}$ & $4.652^{*}$ & $4.582^{*}$ & $4.62^{*}$ \\
\hline Overall Crime Rate ${ }^{b}$ & $87.443^{*}$ & $92.606^{*}$ & $87.656^{*}$ & $86.504^{*}$ \\
\hline CVHTNC & & 0.695 & 0.789 & 0.754 \\
\hline DMMI $^{d}$ & & -1.375 & -1.306 & -1.337 \\
\hline RxPsychotropic & & -0.099 & 0.007 & -0.145 \\
\hline RxHTN & & 2.336 & 2.350 & 2.340 \\
\hline BMI & & $0.288^{*}$ & $0.289^{*}$ & $0.289^{*}$ \\
\hline Current Drug & & & 3.587 & 3.554 \\
\hline Current Alcohol & & & -0.034 & -0.057 \\
\hline Current Smoking & & & -2.056 & -2.123 \\
\hline Negative Affect Componente & & & & 0.391 \\
\hline \multicolumn{5}{|c|}{ 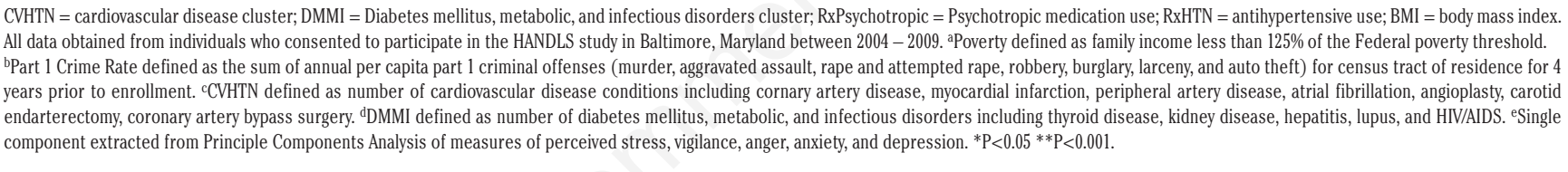 } \\
\hline
\end{tabular}

Table 4. Hierarchical unstandardized regression coefficients for violent crime rate predicting DBP in Black women.

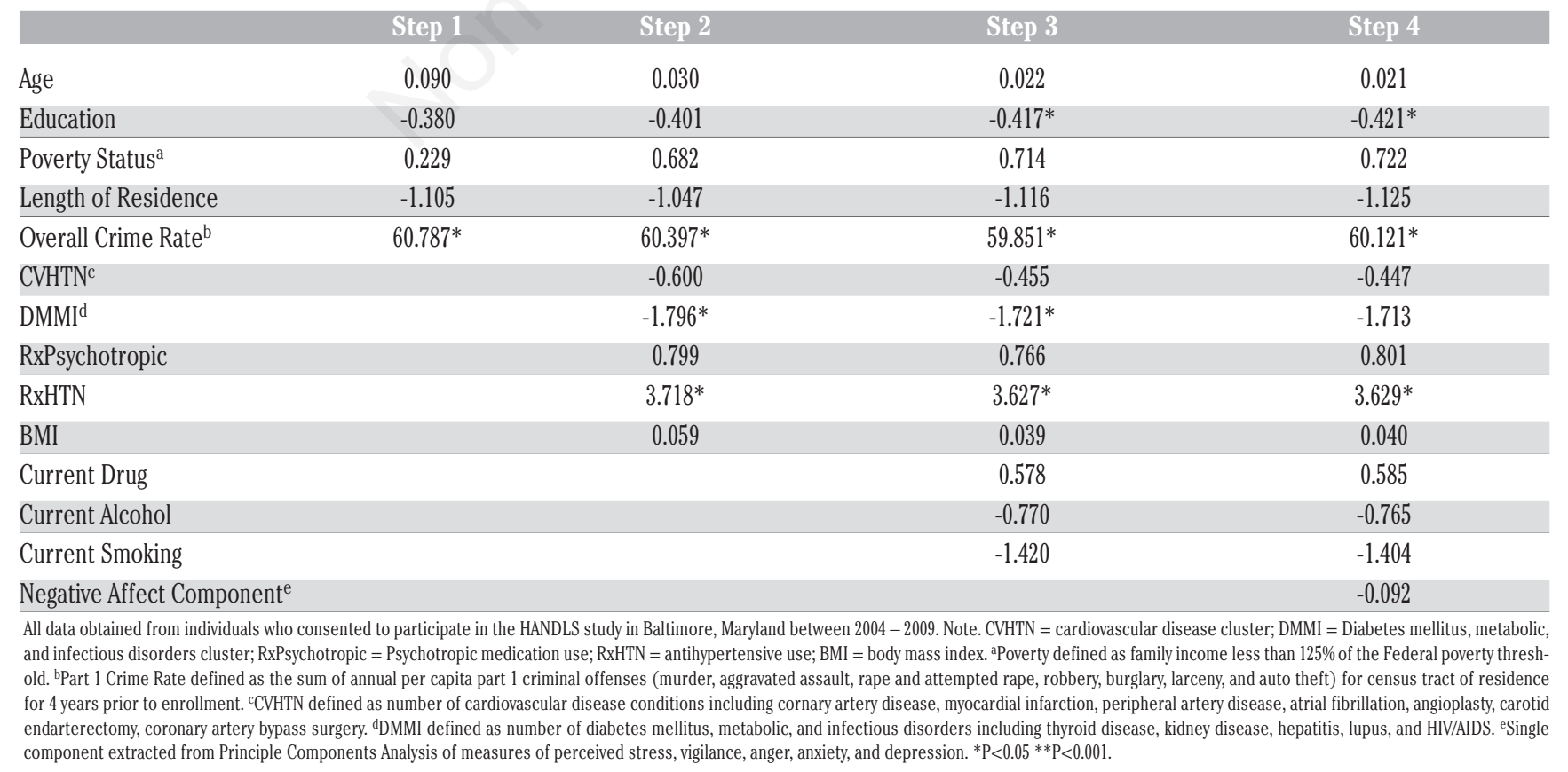


nerable to the health consequences of crime exposure due to their greater concern for personal safety. Thus, a negative response to crime in the form of psychosocial distress might contribute to elevated CVD risk factors among women. ${ }^{32,33,56}$ However, contrary to our hypothesis, the associations between neighborhood crime and CVD risk factors in AA women were not mediated by a negative affect component comprised of measures of perceived stress, vigilance, anger, anxiety, and depression. Although previous findings demonstrated that the association between a composite measure of neighborhood disorder and general health was mediated by depression and anxiety, to our knowledge, no previous investigations examined whether psychosocial factors mediate the relation of neighborhood crime to health outcomes. One reason for this unexpected finding could be that the psychosocial vulnerability component may be too general a construct. Discrete measures of emotionality may be more pertinent to the relation of crime to health outcomes. ${ }^{40,57}$ This will be an important area for future study.

It is unclear why overall crime, and not violent crime, was associated with increased glucose levels and why violent crime, and not overall crime was associated with increased blood pressure. There may be something fundamentally different about the mechanism by which overall crime, which includes violent crime as well as more petty general crimes, affects one's health. Perhaps due to the increased frequency, overall crime represents more of a cumulative, sustained stressor which may be interpreted very differently from a less frequent, more severe stressor such as violent crime. Future research should aim to explore the underlying mechanisms that contribute to the variation in findings.

\section{Study strengths and limitations}

Strengths of this analysis include the large heterogeneous sample offered by the HANDLS study which samples representatively across a range of race, sex, age, and SES combinations. To our knowledge, this is the first study to separately examine participants by race and sex in associations between crime and cardiovascular risk factors. In addition, previous research has often failed to recognize the complex set of individual-level potential confounds thought to drive many of the associations between neighborhood level factors and health outcomes. By including a wide range of individual-level factors, the present study's enhanced attention to the potential role of confounding variables further strengthens confidence in the findings.

The present analysis also has several important limitations, including its cross-sectional design. Future studies should examine prospective associations in order to establish temporal direction. Further, within-census tract variance in exposure to crime may hinder our ability to examine any true associations between prevalence of crime and CVD risk factors. By using objective crime, we assume "exposure" to crime equally within a given census tract. An argument has been made for the use of objective crime because of its resistance to subjective bias, however the perception of crime may drive the association between neighborhood crime and CVD risk factors regardless of its actual prevalence. ${ }^{58}$ In addition, selfreport crime data can be further differentiated into categories reflecting how the crime is experienced such as victimization, witnessing, and hearing about it. ${ }^{16}$ The relative importance of the perception of crime compared to objective crime should be a focus of future research.

The fact that all participants resided in urban inner-city neighborhoods where the prevalence of immeasurable characteristics of the environment may contribute to residual confounding is another limitation. Drug use may be one such factor as drug users tend to be more likely to live in areas of higher crime and tend to have overall metabolic profiles that make them appear seemingly healthier because of appetite suppression. ${ }^{59}$ Given that drug use was a self-report measure in the present study, we may not have an accurate assessment of drug use. Future studies should include more objective measures of drug use and be careful to consider associations between drug use and the variables of interest. Also, the present findings are only generalizable to urban-dwelling AAs and Whites.

Correspondence: Lauren Faulkner, Department of Psychology, University of Maryland, Baltimore County, 1000 Hilltop Circle, Baltimore, MD 21250, USA.

Tel.: +1.434-222-9193

E-mail: faulk1@umbc.edu

Key words: Crime, neighborhood, cardiovascular risk factors, blood pressure, glucose.

Contributions: MRS led conceptualization and design of the present data analyses, performed the statistical analyses, interpreted data, and prepared the manuscript. LMDF participated in data interpretation and manuscript preparation. MKE conceptualized and designed the HANDLS study, participated in interpretation of the present data, and preparation of the manuscript. ABZ conceptualized and designed the HANDLS study, participated in interpretation of the present data, and preparation of the manuscript. SRW supervised conceptualization and design of the present data analyses, participated in interpretation of the present data, and preparation of the manuscript.

Conflict of interests: The authors declare that they have no conflicts of interest.

Funding: This research was support by the National Institute on Aging's Intramural Research Program ZO1-AG000194, and NIH grants RO1 AG034161 and P30 AG028747.

Received for publication: 6 July 2019

Revision received: 6 July 2019.

Accepted for publication: 19 November 2019

@ Copyright: the Author(s), 2019

Licensee PAGEPress, Italy

Journal of Public Health Research 2019;8:1643

doi:10.4081/jphr.2019.1643

This work is licensed under a Creative Commons Attribution NonCommercial 4.0 License (CC BY-NC 4.0).

\section{Conclusions}

Although previous research found associations between neighborhood environment and cardiovascular risk factors, little is known about the specific neighborhood factors that may be contributing to these associations. The present study lays the groundwork for a better understanding of neighborhood effects by revealing several important associations between a specific neighborhood characteristic, objective crime, and CVD risk factors among sociodemographic subgroups known to differ in CVD outcomes. Our findings suggest that crime may be an important characteristic of the neighborhood environment that affects cardiovascular health and may also be an important contributor to differential risk factor associations across race and sex. AA women may be particularly vulnerable to the association between neighborhood crime and cardiovascular risk factors. The results contribute to our understanding of the highly complex multilevel CVD risk factor model, and most importantly, uncover target areas for prevention and intervention. Routine screening for elevated blood pressure and fasting glucose levels amongst AA women living in high crime neighborhoods may help to identify those at greater risk for CVD before 
disease endpoints are reached. Furthermore, preventative efforts aimed toward this group may help to deter the detrimental effects that living in a high crime area may have on one's cardiovascular health. Policies should focus on increasing such efforts within higher crime communities.

\section{References}

1. Roger VL, Go AS, Lloyd-Jones DM, et al. Heart disease and stroke statistics - 2012 update: a report from the American Heart Association. Circulation 2012;125:e2-220.

2. Kaplan G, Keil J. Socioeconomic factors and cardiovascular disease: a review of the literature. Circulation 1993;88:197398.

3. Massey DS. Segregation and stratification: a biosocial perspective. Du Bois Rev 2004;1:7-25.

4. Adler N, Snibbe A. The role of psychosocial processes in explaining the gradient between socioeconomic status and health. Curr Dir Psychol Sci 2003;12:119-23.

5. Chichlowska KL, Rose KM, Diez-Roux A, et al. Individual and neighborhood socioeconomic status characteristics and prevalence of metabolic syndrome: the Atherosclerosis Risk in Communities (ARIC) Study. Psychosom Med 2008;70:98692.

6. Cubbin C, Sundquist K, Ahlén H, Johansson SE, Winkleby MA, Sundquist J. Neighborhood deprivation and cardiovascular disease risk factors: protective and harmful effects. Scand J Public Health 2006;34:228-37.

7. Diez Roux A. Residential environments and cardiovascular risk. J Urban Health 2003;80:569-89.

8. Sundquist K. Neighborhood socioeconomic environment and incidence of coronary heart disease: a follow-up study of 25,319 women and men in Sweden. Am J Epidemiol 2004;159:655-62.

9. Pickett KE, Pearl M. Multilevel analyses of neighbourhood socioeconomic context and health outcomes: a critical review. J Epidemiol Community Health 2001;55:111-22.

10. Cathorall ML, Xin H, Aronson R, et al. The influence of neighborhood poverty on blood glucose levels: findings from the Community Initiative to Eliminate Stroke (CITIES) program. Heal Cult Soc 2015;8.

11. Kershaw KN, Roux AVD, Bertoni A, et al. Associations of chronic individual-level and neighbourhood-level stressors with incident coronary heart disease: the Multi-Ethnic Study of Atherosclerosis. J Epidemiol Community Heal 2014;69:13641.

12. Buys DR, Howard VJ, McClure LA, et al. Association between neighborhood disadvantage and hypertension prevalence, awareness, treatment, and control in older adults: results from the University of Alabama at Birmingham Study of Aging. Am J Public Health 2015;105:1181-8.

13. Sundquist K, Theobald H, Yang M, et al. Neighborhood violent crime and unemployment increase the risk of coronary heart disease: a multilevel study in an urban setting. Soc Sci Med 2006;62:2061-71.

14. Wilson WC, Rosenthal BS. The relationship between exposure to community violence and psychological distress among adolescents: a meta-analysis. Violence Vict 2003;18:335-52.

15. Murali R, Chen E. Exposure to violence and cardiovascular and neuroendocrine measures in adolescents. Ann Behav Med 2005;30:155-63.

16. Wilson DK, Kliewer W, Sica DA. The relationship between exposure to violence and blood pressure mechanisms. Curr
Hypertens Rep 2004;6:321-6.

17. Mobley LR, Root ED, Finkelstein EA, et al. Environment, obesity, and cardiovascular disease risk in low-income women. Am J Prev Med 2006;30:327-32.

18. Liang H, Tomey K, Chen D, et al. Objective measures of neighborhood environment and self-reported physical activity in spinal cord injured men. Arch Phys Med Rehabil 2008;89:1468-73.

19. Wilson D, Kliewer W, Plybon L. Violence exposure and ambulatory blood pressure in African-American adolescents. Int $\mathrm{J}$ Rehabil Heal 1998;4:223-32.

20. Cooley-Quille M, Boyd R. Emotional and behavioral impact of exposure to community violence in inner-city adolescents. J Clin Child Psychol 2001;30:199-206.

21. Clark R, Benkert RA, Flack JM. Violence exposure and optimism predict task-induced changes in blood pressure and pulse rate in a normotensive sample of inner-city black youth. Psychosom Med 2006;68:73-9.

22. Fish JS, Ettner S, Ang A, Brown AF. Association of perceived neighborhood safety on body mass index. Am J Public Health 2010;100:2296-303.

23. Baldock K, Paquet C, Howard N, et al. Associations between resident perceptions of the local residential environment and metabolic syndrome. J Environ Public Health 2012. doi:10.1155/2012/589409.

24. Browning CR, Cagney KA, Iveniuk J. Neighborhood stressors and cardiovascular health: crime and C-reactive protein in Dallas, USA. Soc Sci Med 2012;75:1271-9.

25. Wilson DK, Kliewer W, Teasley N, et al. Violence exposure, catecholamine excretion, and blood pressure nondipping status in African American male versus female adolescents. Psychosom Med 2002;64:906-15.

26. Smalls BL, Gregory CM, Zoller JS, Egede LE. Assessing the relationship between neighborhood factors and diabetes related health outcomes and self-care behaviors. BMC Health Serv Res 2015;15:445.

27. Wang MC, Kim S, Gonzalez AA, et al. Socioeconomic and food-related physical characteristics of the neighbourhood environment are associated with body mass index. J Epidemiol Community Health 2007;61:491-8.

28. Mathis AL, Rooks RN, Tawk RH, Kruger DJ. Neighborhood influences and BMI in urban older adults. J Appl Gerontol 2017;36:692-708.

29. Agyemang C, van Hooijdonk C, Wendel-Vos W, et al. Ethnic differences in the effect of environmental stressors on blood pressure and hypertension in the Netherlands. BMC Public Health 2007;7.

30. Chum A, O'Campo P. Cross-sectional associations between residential environmental exposures and cardiovascular diseases. BMC Public Health 2015;15.

31. Glass TA, Rasmussen MD, Schwartz BS. Neighborhoods and obesity in older adults: the Baltimore Memory Study. Am J Prev Med 2006;31:455-63.

32. Hale C. Fear of crime: a review of the literature. Int Rev Vict 1996;4:79-150

33. Foster S, Giles-Corti B. The built environment, neighborhood crime and constrained physical activity: an exploration of inconsistent findings. Prev Med (Baltim) 2008;47:241-51.

34. Schulz AJ, Williams DR, Israel BA, Lempert LB. Racial and spatial relations as fundamental determinants of health in Detroit. Milbank Q 2002;80:677-707.

35. Gillum R. The epidemiology of cardiovascular disease in black Americans. N Engl J Med 1996;335:1597-9.

36. Mujahid M, Roux A, Cooper R, Shea S, Williams D. 
Neighborhood stressors and race/ethnic differences in hypertension prevalence (the Multi-Ethnic Study of Atherosclerosis). Am J Hypertens 2011;24:187-93.

37. Flack JM, Ferdinand KC, Nasser SA. Epidemiology of hypertension and cardiovascular disease in African Americans. J Clin Hypertens (Greenwich). 2003;5:5-11.

38. Inoue Y, Stickley A, Yazawa A, et al. Neighborhood characteristics and cardiovascular risk among older people in Japan: findings from the JAGES project. PloS One 2016;11:e0164525.

39. Koopman C, Van Oeffelen AA, Bots ML, et al. Neighbourhood socioeconomic inequalities in incidence of acute myocardial infarction: a cohort study quantifying age- and gender- specific differences in relative and absolute terms. BMC Public Health 2012;12:617.

40. Gallo LC, Matthews KA. Understanding the association between socioeconomic status and physical health: do negative emotions play a role? Psychol Bull 2003;129:10-51.

41. Hill T, Ross C, Angel R. Neighborhood disorder, psychophysiological distress, and health. J Health Soc Behav 2005;46:170-86.

42. Lynch JW, Kaplan GA, Cohen RD, Tuomilehto J, Salonen JT. Do cardiovascular risk factors explain the relation between socioeconomic status, risk of all-cause mortality, cardiovascular mortality, and acute myocardial infarction? Am J Epidemiol 1996;144:934-42.

43. Schulz AJ, Kannan S, Dvonch JT, et al. Social and physical environments and disparities in risk for cardiovascular disease: the Healthy Environments Partnership Conceptual model. Environ Health Perspect 2005; 113:1817-25.

44. Gump BB, Matthews KA, Räikkönen K. Modeling relationships among socioeconomic status, hostility, cardiovascular reactivity, and left ventricular mass in African American and White children. Health Psychol 1999;18:140-50.

45. Ruberman W, Weinblatt E, Goldberg J, Chaudhary B. Psychosocial influences on mortality after myocardial infarction. N Engl J Med 1984;311:552-9.

46. Ickovics JR, Viscoli CM, Horwitz RI. Functional recovery after myocardial infarction in men: the independent effects of social class. Ann Intern Med 1997;127:518-25.

47. Evans M, Lepkowski J, Powe N, et al. Healthy aging in neigh- borhoods of diversity across the life span (HANDLS): overcoming barriers to implementing a longitudinal, epidemiologic, urban study of health, race, and socioeconomic status. Ethn Dis 2010;20:267-75.

48. Cohen S, Kamarck T, Mermelstein R. A global measure of perceived stress. J Health Soc Behav 1983;24:385-96.

49. Radloff L. The CES-D scale A self-report depression scale for research in the general population. Appl Psychol Meas 1977;1:385-401.

50. Zimmerman M, Mattia J. A self-report scale to help make psychiatric diagnoses: the Psychiatric Diagnostic Screening Questionnaire. Arch Gen Psychiatry 2001;58:787-94.

51. Taylor SE, Seeman TE. Psychosocial resources and the SEShealth relationship. Ann N Y Acad Sci. 1999;896:210-25.

52. Buss a H, Perry M. The aggression questionnaire. J Pers Soc Psychol 1992;63:452-9.

53. Rozanski A, Blumenthal JA, Kaplan J. Impact of psychological factors on the pathogenesis of cardiovascular disease and implications for therapy. Circulation 1999;99:2192-217.

54. Guttman L. Some necessary conditions for common-factor analysis. Psychometrika 1954;19:149-61.

55. Mendall M, Patel P, Ballam L. C reactive protein and its relation to cardiovascular risk factors: A population based cross sectional study. BMJ 1996;312:1061-5.

56. Unger E, Diez-Roux AV, Lloyd-Jones DM, et al. Association of neighborhood characteristics with cardiovascular health in the multi-ethnic study of atherosclerosis. Circ Cardiovasc Qual Outcomes 2014;7:524-31.

57. Suls J, Bunde J. Anger, anxiety, and depression as risk factors for cardiovascular disease: the problems and implications of overlapping affective dispositions. Psychol Bull 2005;131: 260-300.

58. Taylor R, Shumaker S. Local crime as a natural hazard: Implications for understanding the relationship between disorder and fear of crime. Am J Community Psychol 1990;18:61941.

59. Debeck K, Wood E, Zhang R, et al. A dose-dependent relationship between exposure to a street-based drug scene and healthrelated harms among people who use injection drugs. J Urban Health 2011;88:724-35. 